\title{
Ascites symptom inventory-7 is a valuable tool for evaluating the effectiveness of tolvaptan in patients with cirrhotic ascites
}

\author{
HIDETO KAWARATANI, KEI MORIYA, TADASHI NAMISAKI, NAOTAKA SHIMOZATO, KOSUKE KAJI, \\ HIROAKI TAKAYA, YUKIHISA FUJINAGA, YASUHIKO SAWADA, SHINYA SATO, SOICHIRO SAIKAWA, \\ TAKUYA KUBO, TAKEMI AKAHANE, HIROSHI FUKUI and HITOSHI YOSHIJI
}

Third Department of Internal Medicine, Nara Medical University, Kashihara, Nara 634-8522, Japan

Received July 3, 2019; Accepted September 8, 2020

DOI: $10.3892 / \mathrm{etm} .2020 .9462$

\begin{abstract}
Patients with liver cirrhosis frequently experience non-specific symptoms and report severe reductions in their quality of life (QOL). The underlying mechanisms of the disease are multifactorial that may be specific to the disease or directly related to the liver. The major concern of liver cirrhosis with ascites, however, is the decreased QOL. Therefore, in the present study, the Ascites Symptom Inventory-7 (ASI-7) questionnaire was applied to subjectively evaluate the symptoms in patients with cirrhotic ascites following tolvaptan administration. In total, 69 patients with liver cirrhosis with ascites hospitalized to Nara Medical University were evaluated after being treated with tolvaptan (3.75-7.5 mg/day) and conventional diuretics between December 2013 and April 2018. A follow-up assessment was conducted 7 days after tolvaptan treatment, whilst ASI-7 was used on days 1 and 8 of the study. After an uneventful 7-day tolvaptan treatment regimens, 49 patients $(71.0 \%)$ lost $>1.5 \mathrm{~kg}$ of their body weight, who were referred to as responders, with the change in the ASI-7 score being found to correlate with the body weight change. By contrast, changes in urine volume did not correlate with those in the ASI-7 score. The responders experienced a greater reduction in the ASI-7 score after 7 days compared with those in the non-responders $(\mathrm{P}<0.01)$. ASI-7 scores were also found to correlate with body weight after tolvaptan administration. In conclusion, ASI-7 accurately reflected changes in body weight but not urine volume and results of the study highlighted the value of ASI-7 in the evaluation of ascitic volume and effectiveness of tolvaptan in cirrhotic ascites. The present clinical trial was registered onto the UMIN-Clinical Trial Registry on 1st March 2014 (registration no. UMIN000013095).
\end{abstract}

Correspondence to: Dr Hideto Kawaratani, Third Department of Internal Medicine, Nara Medical University, 840 Shijo-cho, Kashihara, Nara 634-8522, Japan

E-mail: kawara@naramed-u.ac.jp

Key words: ascites symptom inventory-7, cirrhotic ascites, tolvaptan, quality of life, patient-reported outcome

\section{Introduction}

Patients with liver cirrhosis frequently experience non-specific symptoms and report a severe reduction in their quality of life (QOL) $(1,2)$. Patient-reported outcome (PRO) is an outcome-reporting system directly reported by the patient to describe their own experience of the condition, which differs from that reported by physicians and nurses (3). PRO method, similar to questionnaires, is used in clinical trials to gain a better understanding of the efficacy or effectiveness of a treatment modality (4). There are several questionnaires concerning liver disease, including the Chronic Liver Disease (CLD) Questionnaire (CLD-Q) (5), Liver Disease Quality of Life Questionnaire (6) and the Liver Disease Symptom Index 2.0 (LDSI 2.0) (7), which are designated to assess the QOL of patients with CLD. Although the CLD-Q and LDSI 2.0 contain questions regarding abdominal symptoms, their primary aim is not to assess the symptoms of ascites. By contrast, Ascites Symptom Inventory-7 (ASI-7) is specific to ascites symptoms, which directly reflects the abdominal symptoms of the patients (8). The effectiveness of ASI-7 has been previously reported (9), though the existence of the questionnaire in not widely known.

In a previous study of ascending levels of tolvaptan (SALT) in 2006, an orally active vasopressin V2-receptor antagonist, tolvaptan, was first reported as safe and effective for increasing serum sodium concentrations among patients with hyponatremia $(9,10)$. Since December 2013, tolvaptan has been used in Japan owing to a mechanism of action that is different from those of conventional diuretics, including furosemide or spironolactone (11). Tolvaptan acts by suppressing the expression of Aquaporin (AQP)-2, inhibiting water reabsorption in the renal collecting ducts (12). Unlike other diuretics, this drug does not stimulate sodium channels; instead, it increases free water excretion without affecting urinary sodium levels (13).

A number of reports have previously assessed the QOL of patients with refractory ascites $(14,15)$. In addition, there is an insufficient number of assessments on the effectiveness of ASI-7 following the administration of tolvaptan in treating cirrhotic ascites, where decreases in ascites may reduce the ASI-7 score (8). Therefore, the aim of the present cohort study was to apply the ASI-7 questionnaire to evaluate the symptoms subjectively after tolvaptan administration in patients with cirrhotic ascites. 


\section{Materials and methods}

Therapeutic protocol. The protocol was registered to the clinical trials registry managed by the University Hospital Medical Information Network in Japan (registration no. UMIN000013095). This trial was conducted between December 2013 and April 2018 and consisted of patients with liver cirrhosis accompanied by ascites who fulfilled the following criteria in accordance with a Japanese phase III study (11): (i) $>20$ years of age; and (ii) experienced persistent ascites despite conventional diuretic treatments with $\geq 20 \mathrm{mg} /$ day furosemide and/or $\geq 25 \mathrm{mg} /$ day spironolactone. The following exclusion criteria were adopted from a phase III Japanese study (11): i) Having other complications or malignancies such as hepatic encephalopathy (West haven criteria $\geq$ grade II) (16); ii) vascular invasive hepatocellular carcinoma, esophageal or gastric varices requiring new treatment, repeated hemorrhoid bleeding due to rectal varices, congestive heart failure and anuria or impaired urination (10); (iii) a history of cerebrovascular disorders; (iv) hemoglobin levels $<8.0 \mathrm{~g} / \mathrm{dl}$, total serum sodium $<120$ or $>147 \mathrm{mEq} / \mathrm{l}$ or serum potassium $>5.5 \mathrm{mEq} / \mathrm{l}$; (v) inability to take oral medication; and (vi) patients otherwise adjudged by the investigator to be inappropriate for inclusion into the study.

The present study consisted of a 2-day pretreatment observation period and a 7-day treatment period. All patients received 3.75 or $7.5 \mathrm{mg} /$ day tolvaptan for 7 days as an adjunct to conventional diuretics $(10-60 \mathrm{mg}$ /day furosemide and/or $25-100 \mathrm{mg} /$ day spironolactone) during sodium restriction ( $<7 \mathrm{~g} /$ day). Day 1 was defined as the first day of tolvaptan administration. Data obtained immediately before the administration of tolvaptan were used as baseline data. Day 8 was defined as the end of the tolvaptan treatment. The primary outcome of this study is to investigate if body weight loss correlates with improvements in the ASI-7 scores following tolvaptan administration, whilst the secondary outcome is to assess the relationship between the ASI-7 score and parameters such as urine volume or hepatic reserve.

Patientenrollment. In this prospective, single-arm, multi-center study, a total of 116 consecutive patients with cirrhotic ascites treated with tolvaptan were enrolled. However, only 80 patients completed the ASI-7 questionnaire. Patients that underwent paracentesis, experienced hepatic coma during the treatment period or submitted questionnaires with missing data were excluded. In total, 11 patients dropped out of the study due to various reasons (Fig. 1). Finally, a total of 69 patients were included for the analysis. The clinical characteristics of the patients with cirrhotic ascites are shown in Table I.

Ethics. The present study was approved by the Institutional Review Board (IRB) of Nara Medical University (IRB approval no. 2-9). This study was conducted in accordance with the ethical standards in the Declaration of Helsinki. Written informed consent was obtained from all patients prior to their participation in the study.

Physical examination findings. The body weight (BW) and urine output of each participant were measured every day of the study. Consistent with previous reports (17), responders to the
Table I. Clinical characteristics of patients.

\begin{tabular}{lc}
\hline Parameter & Data $^{\mathrm{a}}$ \\
\hline Age (years) & $68.8 \pm 11.5$ \\
Sex (male/female) & $43 / 26$ \\
Cause of cirrhosis (HBV/HCV/Alc/others) & $7 / 24 / 17 / 21$ \\
Ascites volume (mild/moderate/severe) & $10 / 25 / 34$ \\
Child-Pugh classification (B/C) & $33 / 36$ \\
Child-Pugh score & $10.1 \pm 1.7$ \\
Dose of tolvaptan received (3.75/7.5 mg) & $7 / 62$ \\
Dose of furosemide (mg) & $25.6 \pm 11.7$ \\
Dose of spironolactone (mg) & $50.9 \pm 16.2$ \\
With HCC & $29(42.0 \%)$ \\
With portal vein thrombus & $18(26.1 \%)$ \\
\hline
\end{tabular}

aPresented either as the mean $\pm \mathrm{SD}, \mathrm{N}$, or $\mathrm{N}(\%)$. HBV, hepatitis B virus; $\mathrm{HCV}$, hepatitis C virus; alc, alcoholic; $\mathrm{HCC}$, hepatocellular carcinoma.

Table II. Items in the ASI-7 questionnaire ${ }^{\mathrm{a}}$.

\begin{tabular}{ll}
\hline Number & \multicolumn{1}{c}{ Item } \\
\hline 1 & My stomach feels heavy. \\
2 & My stomach is bloated and I feel discomfort. \\
3 & My stomach is bloated, so it is difficult \\
& to move around. \\
4 & My stomach feels heavy when lying down. \\
5 & I have difficulty breathing after walking \\
6 & for 2-3 min. \\
7 & I cannot eat because my stomach is bloated. \\
& I cannot take deep breaths.
\end{tabular}

anstructions: 'Please answer the following questions about your ascites (abdominal fluid or fullness) and related symptoms, as they are today'. Likert Scale: i) 0, does not apply at all; ii) applies slightly; iii) applies somewhat; iv) applies strongly; and v) applies very strongly. ASI-7, ascites symptom inventory-7.

treatment were defined as patients exhibiting $>1.5-\mathrm{kg}$ loss in BW after 1-week tolvaptan administration whereas non-responders were defined as those having exhibiting weight loss $<1.5 \mathrm{~kg}$ or weight gain. Child-Pugh scores were calculated from the five clinical measures of liver disease. Each measure was allocated a score of 1-3, with 3 indicating severe derangement. The five clinical measures were as follows: Total bilirubin, serum albumin, prothrombin time, ascites and hepatic encephalopathy (18).

Questionnaire. The ASI-7 was used on days 1 and 8 of the study. It consists of seven items on a 5-point Likert scale: 0 , does not apply; 1 , slightly applies; 2 , somewhat applies; 3 , strongly applies; and 4 , very strongly applies. The following was written on each questionnaire: 'Please answer the following questions about your ascites (abdominal fluid or fullness) and related symptoms, as they are today'. The seven items comprising the scale shown in Table II cover various 
domains and were arranged in a sequence from mild to severe symptoms, facilitating clinical interpretation. The cumulative score was calculated by summing up the scores of each of the 7 items (0-4 points), which can range from 0 to 28 points. The scale of the ASI-7 scoring system showed asymmetric distribution within a range of $0-28$ points. The score system of ASI-7 was defined as follows: i) Slight ascites, 0-11; ii) mild ascites, 12-18; iii) moderate ascites, 19-22; and iv) severe ascites, 23-28. $\triangle \mathrm{ASI}-7$ scores were defined as 'ASI-7 score on day 8', 'ASI-7 score on day 1'.

Statistical analysis. Patient backgrounds and clinical parameters are presented as the number of patients (\%) or means \pm SD. Mann-Whitney U test was used for comparisons between two groups. Kruskal-Wallis test followed by Dunn's test was performed for comparisons among three groups. Spearman's rank correlation analysis was performed to determine the correlation coefficient (R) and P-value. All statistical analyses were performed using SPSS (SPSS, Inc.) for Windows version 16.0. $\mathrm{P}<0.05$ were considered to indicate a statistically significant difference.

\section{Results}

Baseline characteristics of patients. In total, 69 patients with difficult-to-treat ascites were analyzed. Table I shows the baseline (day 1) clinical characteristics of the patients enrolled. All enrolled patients had liver cirrhosis, with the mean age of $68.8 \pm 11.5$ years (range: $34-85$ years); 43 patients $(62.3 \%)$ were male and 26 patients $(37.7 \%)$ were female. In total, 60 patients $(87.0 \%)$ received furosemide (mean dose, $25.6 \pm 11.7 \mathrm{mg} /$ day; range, $10-60 \mathrm{mg} /$ day) and 62 patients $(89.9 \%)$ received spironolactone (mean dose, $50.9 \pm 16.2 \mathrm{mg} /$ day; range: $25-100 \mathrm{mg} /$ day). Of the 69 patients, 62 received $7.5 \mathrm{mg}$ tolvaptan, whilst the remaining 7 received $3.75 \mathrm{mg}$ tolvaptan. The cause of liver cirrhosis in the participants were as follows: i) 7 patients had hepatitis B; ii) 24 patients had hepatitis C; iii) 17 patients had a history of alcoholism; and iv) 21 patients had other disorders, including primary biliary cholangitis and autoimmune hepatitis. In total, 33 patients had Child-Pugh grade B and 36 patients had Child-Pugh grade C. Of the 69 participants, 18 patients $(26.1 \%)$ had portal vein thrombus and $29(42.0 \%)$ had complications with hepatocellular carcinoma; these patients but did not experience invasion of the portal vein. The amount of ascites was classified into the mild, moderate and severe groups, proposed by the International Club of Ascites (19), as estimated using CT or ultrasonography (US). In total, 10 patients had mild ascites, 25 patients had moderate ascites, and the remaining 34 patients had severe ascites.

Correlation among each baseline parameter. Baseline ASI-7 score was first obtained on day 1, where mean score of 13.7 \pm 8.1 was documented and the ASI-7 score increased with ascites volume. For patients with mild, moderate and severe ascites, the median ASI-7 scores were found to be 6, 13 and 15 respectively (Fig. 2). Comparison of the three groups revealed a significant difference by Kruskal-Wallis test $(\mathrm{P}=0.02)$. The ASI-7 scores of patients with severe ascites were significantly higher compared with those of patients with mild

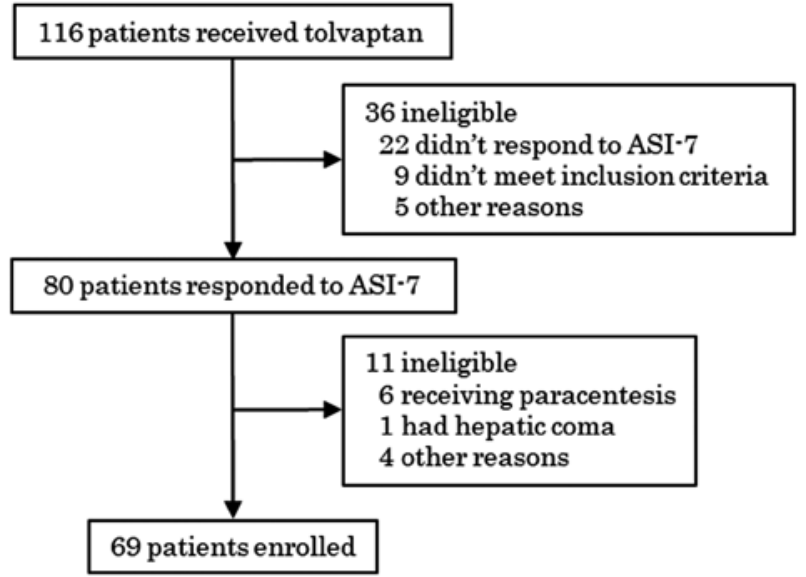

Figure 1. Study work flow. ASI-7, ascites symptom inventory-7.

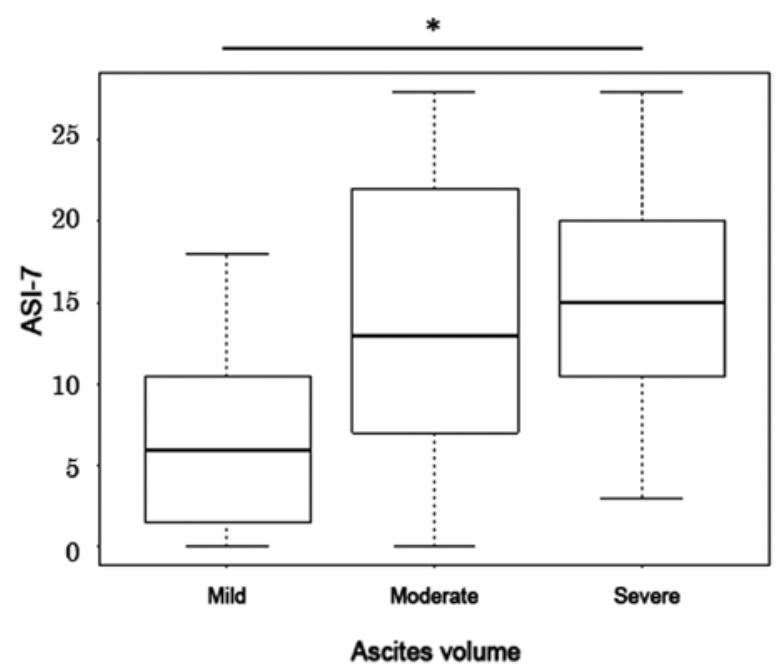

Figure 2. Median ASI-7 scores in patients with mild, moderate and severe ascites. ${ }^{*} \mathrm{P}<0.05$. ASI-7, ascites symptom inventory-7.

ascites ( $\mathrm{P}=0.01$; Fig. 2). There were no difference between moderate vs. mild and moderate vs. severe. Additionally, there was no correlation between the Child-Pugh and ASI-7 scores between day 8 and day 1 ( $r<0.001$; Fig. 3$)$.

Effectiveness of 7-day treatment with tolvaptan. In total, 49 patients $(71.0 \%)$ experienced a reduction in $\mathrm{BW}(>1.5 \mathrm{~kg})$ after 7 days, with 32 patients (46.4\%) experiencing $>500-\mathrm{ml}$ increase in urine volume the day 1 after tolvaptan treatment. The remaining participants experienced a decrease in urine volume. Correlation between BW change in 7 days and changes in urine volume the day 1 after tolvaptan treatment was assessed, A weak but insignificant correlation between changes in BW and urine volume was observed ( $\mathrm{r}=-0.079$; Fig. 4). The mean ASI-7 scores also decreased from $13(\mathrm{Q} 3-\mathrm{Q} 1: 20-6.5)$ to 5 (Q3-Q1:11-0) after 7 days of treatment. Correlation between the changes in the BW and ASI-7 scores in 7 days was next assessed, where a moderate and significant correlation between the BW change in 7 days and ASI-7 score change ( $\mathrm{r}=0.524 ; \mathrm{P}<0.01$; Fig. 5) was noted. By contrast, no correlation was observed between the BW change after 7 days and change of urine volume at day 1 (data 


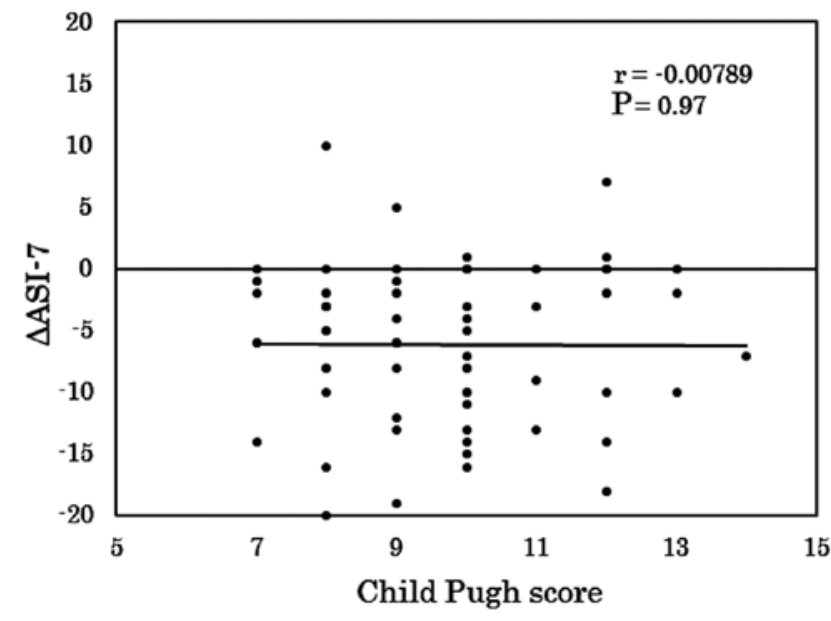

Figure 3. Correlation between the Child-Pugh score and $\Delta$ ASI-7. No correlation between Child-Pugh score and weight change was noted. ASI-7, ascites symptom inventory-7.

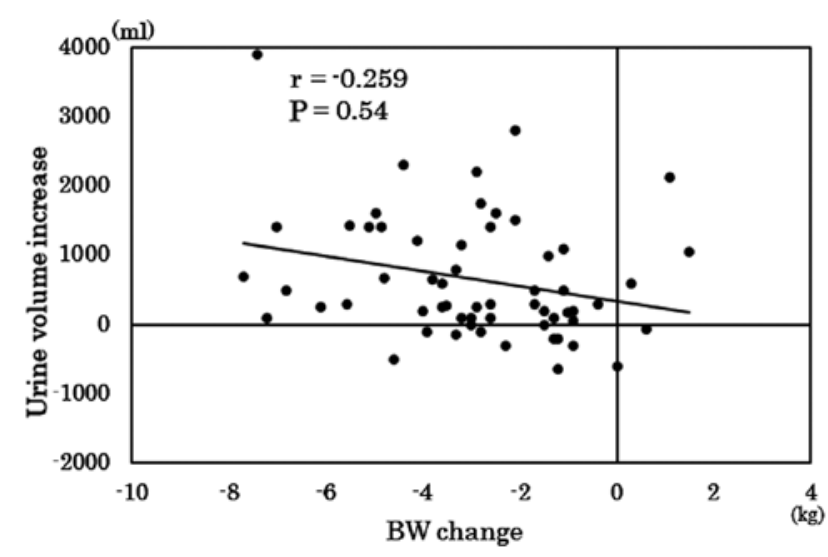

Figure 4. Correlation between urine output increase and subsequent body weight change. A small correlation between urine volume increase and weight change was noted. BW, body weight.

not shown; $\mathrm{r}=0.137 ; \mathrm{P}=0.15)$. The treatment response was evaluated on the basis of changes in the BW after tolvaptan treatment. Among the responders, $43 / 49$ patients $(87.8 \%$ ) experienced reduced ASI-7, whilst 13/20 non-responder patients (65.0\%) had decreased ASI-7. Responders and non-responders had an median ASI-7 score change of -6 (Q3-Q1:-11.5--2) and -3 (Q3-Q1:-5--2), respectively, with a significant difference reported ( $\mathrm{P}=0.012$; Fig. 6).

Consideration of each parameter of ASI-7. Correlation between the $\Delta$ ASI-7 in each item and changes in BW is shown in Fig. 7. The strongest correlation was observed between item no. 2, which stated 'my stomach is bloated and uncomfortable' and changes in $\mathrm{BW}(\mathrm{r}=0.485 ; \mathrm{P}<0.01$; Fig. 7B). Item no. 3, which stated 'my stomach is bloated, so it is difficult to move around', also exhibited a second most notable correlation with changes in $\mathrm{BW}(\mathrm{r}=0.463 ; \mathrm{P}<0.01$; Fig. 7C). Item 7, which stated 'I cannot take deep breaths' exhibited a third most notable correlation with changes in $\mathrm{BW}(\mathrm{r}=0.286$; $\mathrm{P}<0.05$; Fig. $7 \mathrm{G})$. The $\Delta$ ASI-7 of the remaining items (nos. 1, 4, 5, 6 and 7) had either weak or no correlation with changes in BW.

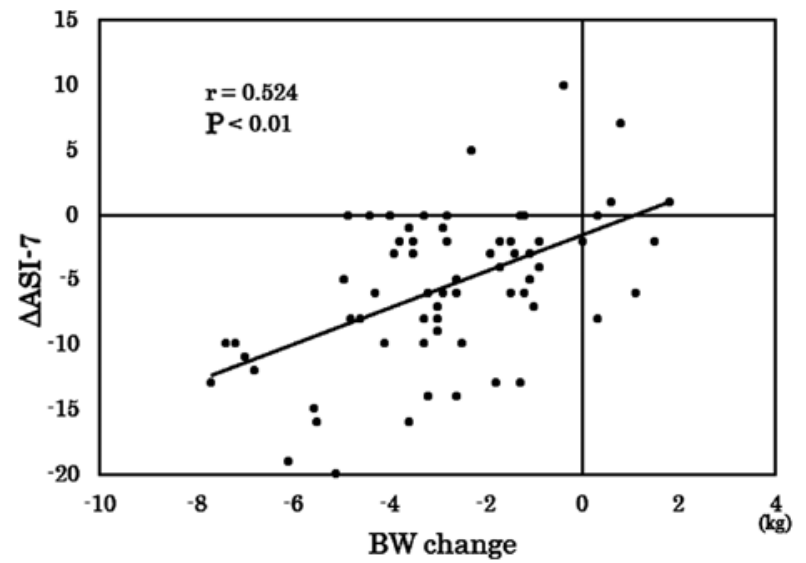

Figure 5. Correlation between ASI-7 score alteration and subsequent body weight change. A moderate correlation between changes in the ASI-7 score and body weight change was noted. $\mathrm{P}<0.01$. ASI-7, ascites symptom inventory-7; BW, body weight.

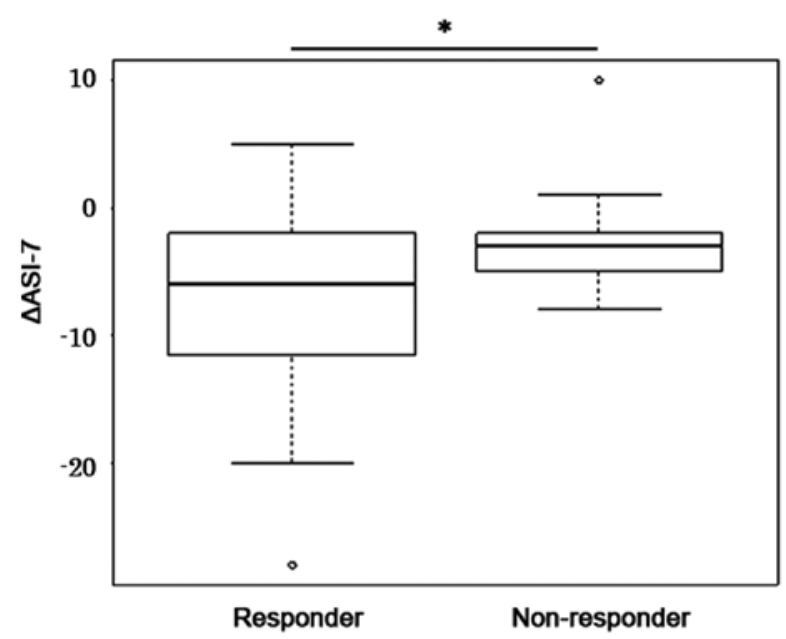

Figure 6. Median changes in the ASI-7 scores in responders and non-responders. " $\mathrm{P}<0.05$. ASI-7, ascites symptom inventory-7.

\section{Discussion}

The results from the present cohort study demonstrated that following 7 days tolvaptan treatment, responders lost weight, such that changes in the their ASI-7 scores correlated with changes in BW. However, changes in urine volume did not correlate with the changes in the ASI-7 scores. Responders showed a further decrease in the ASI-7 scores after 7 days compared with those in non-responders.

ASI-7 is primarily focused on the symptoms related to the ascites of the patients. Only two reports have previously discussed the use of ASI-7 in assessing the symptoms and wellbeing of patients with ascites $(8,15)$. Objective parameters, including BW, urine volume, abdominal circumference and ascites volume measured using US or CT, were measured before and after tolvaptan treatment. The primary goal of ascites treatment is to relieve the symptoms suffered by the patients and consequently improve the QOL (20). The present study was the first to use ASI-7 in evaluating cirrhotic patients with ascites who have been treated with tolvaptan, which confirmed that the ASI-7 is a valuable tool for assessing the 

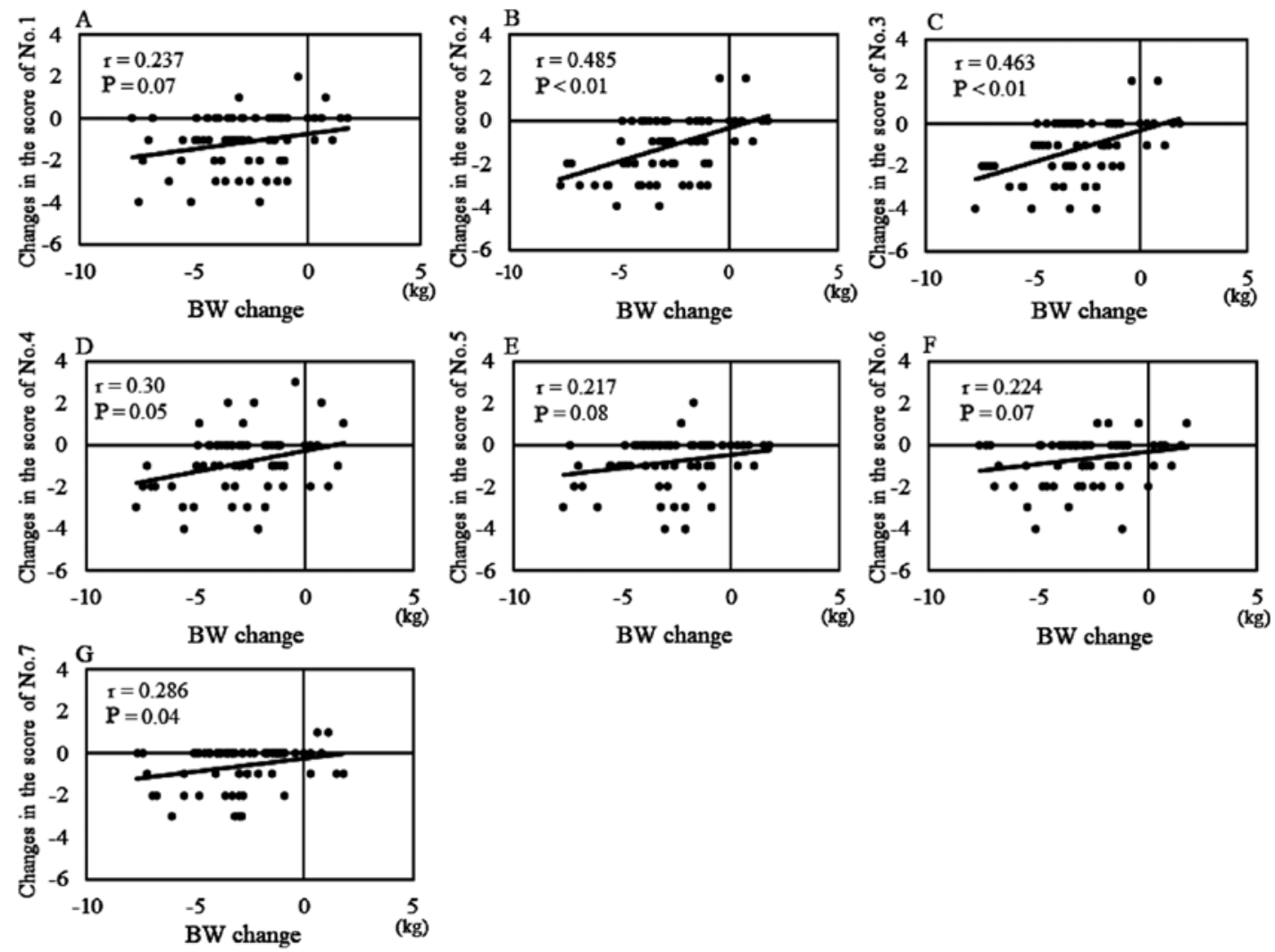

Figure 7. Correlation between changes in the scores of each of the seven items of ASI-7 and BW change. Correlation between changes in the ASI-7 scores of items (A) no. 1, (B) no. 2, (C) no. 3, (D) no. 4, (E) no. 5, (F) no. 6 and (G) no. 7 and changes in body weight. ASI-7, ascites symptom inventory-7; BW, body weight.

response to and effectiveness of tolvaptan administration after a 1-week study period.

Findings from the present study demonstrated that hepatic reserves (Child-Pugh score) did not correlate with the ASI-7 score. Generally, patients with severe ascites have high ASI-7 scores (8). In the present study, an elevation in the ASI-7 score was found to associate with an increase in the ascitic volume. In total, 4 of the 10 patients with mild ascites had severe hepatic hydrothorax with most having high ASI-7 scores, which may have influenced the severity of hepatic hydrothorax. However, some patients with severe ascites had low ASI-7 scores. This may be attributed to the presence of ascites for an extended period of time with only few abdominal symptoms. A dispersion is therefore observed between the volume of ascites and ASI-7 scores, which may be affected by the length of time the patient has had severe ascites by extension of the abdominal wall. To the best of our knowledge, however, there is no previous studies reporting the extension of the abdominal wall caused by the distension of severe ascites for a few weeks.

In patients with liver cirrhosis, the intrahepatic resistance is increased by disruption in the intrahepatic blood flow (21). Consequently, the renin-angiotensin-aldosterone system, sympathetic nervous system and antidiuretic hormone are activated, thereby reducing renal blood flow, sodium and water reabsorption, leading to the development of ascites and edema (22). The most effective treatment for ascites, other than diuretics, is the transjugular intrahepatic portosystemic shunt procedure (23) or large-volume paracentesis (24), which also improve the QOL of the patients. A reduction in BW instead of an increase in urine output has been used as the primary endpoint in clinical trials with tolvaptan (25). This is due to BW changes being considered to be the clinically more important parameter, since they can accurately reflect the volume overload in patients with ascites (26). In the present study, a weak correlation was noted between BW change and urine output increase, suggesting that the increase in urine output does not always reflect BW change. The phase III trial showed an average decrease of $\sim 2.0 \mathrm{~kg} /$ week in BW (11). However, a recent report concluded that a $1.5-\mathrm{kg}$ decrease in 7 days was the best cut-off value in patients with hepatic edema receiving tolvaptan treatment, as symptom reduction was achieved (17). A responder was therefore defined in the study as those who experienced a $1.5-\mathrm{kg}$ decrease in BW in 7 days. Patients who were tolvaptan responders had an $87.8 \%$ decrease in the ASI-7 score whereas patients termed non-responders experienced a $65.0 \%$ decrease in the ASI-7 score. A significant difference was observed between responders and non-responders, suggesting that tolvaptan served an important role in the reduction in the ASI-7 scores. There was a significant correlation between the BW change and the ASI-7 score change. Responders to tolvaptan had an average ASI-7 score change of -7.8, whilst non-responders exhibited an average change in the ASI-7 score of -2.8 , meaning that some patients showed a decrease in the ASI-7 score without a concomitant BW decrease. Although the reasons for this are highly complex, some cases could have been affected by the mental impact. Patients who experienced an increase in urine output without a reduction in BW occasionally feel an improvement in their symptoms associated with ascites. Additionally, results from the present study suggest that assessments using the ASI-7 items 2 and 3 may accurately 
reflect the impact on abdominal symptoms. These observations suggest that changes in the ASI-7 score is reflected by a reduction in patient symptoms after 7 days of tolvaptan treatment and item 2 of the ASI-7 scoring system was the most influential item for indicating ascites symptoms.

There is a number of limitations associated with the present study. They include the small number of patients and utilization of only one type of questionnaire, ASI-7. Since there are other questionnaires, including the SF-36, CLD-Q, or LDSI 2.0, difficulty remains in concluding ASI-7 to be the most useful tool for assessing tolvaptan treatment. However, as other questionnaires do not target ascites, using the ASI-7 appeared to be more useful in assessing the symptoms of ascites. The present study was also limited in that all patients enrolled were Japanese. It has been previously reported that the health-related quality of life patterns differs between Asian and Western populations owing to several different patient-reported outcomes (27).

In conclusion, the present study confirmed the potential use of the ASI-7 questionnaire as an effective tool for assessing patients with hepatic ascites. The majority of patients who responded to treatment with tolvaptan also exhibited a reduction in the ASI-7 score after 7 days of tolvaptan administration, which may serve as a reflection of BW change. ASI-7 has proven to be a valuable tool for assessing information, such as the abdominal symptoms associated with cirrhotic ascites.

\section{Acknowledgements}

Not applicable.

\section{Funding}

No funding was received.

\section{Availability of data and materials}

The datasets used and/or analyzed during the current study are available from the corresponding author on reasonable request.

\section{Authors' contributions}

HK, NS, KK, HT, YF, SSat, SSai and TK collected the data. $\mathrm{KM}, \mathrm{TN}$ and YS analyzed the data. HK and TA interpreted the data. HF and HY contributed to the design of the work. All authors read and approved the final manuscript.

\section{Ethics approval and consent to participate}

The present study was approved by the Institutional Review Board of Nara Medical University. Written informed consents were obtained for the present study.

\section{Patient consent for publication}

Not applicable.

\section{Competing interests}

The authors declare that they have no competing interests.

\section{References}

1. Bhanji RA, Carey EJ and Watt KD: Review article: Maximising quality of life while aspiring for quantity of life in end-stage liver disease. Aliment Pharmacol Ther 46: 16-25, 2017.

2. Marchesini G, Bianchi G, Amodio P, Salerno F, Merli M, Panella C, Loguercio C, Apolone G, Niero M, et al: Factors associated with poor health-related quality of life of patients with cirrhosis. Gastroenterology 120:170-178, 2001.

3. Studenic P, Radner H, Smolen JS and Aletaha D: Discrepancies between patients and physicians in their perceptions of rheumatoid arthritis disease activity. Arthritis Rheum 64: 2814-2823, 2012.

4. Gutteling JJ, de Man RA, van der Plas SM, Schalm SW, Busschbach JJ and Darlington AS: Determinants of quality of life in chronic liver patients. Aliment Pharmacol Ther 23: 1629-1635, 2006.

5. Prinsen CA, Mokkink LB, Bouter LM, Alonso J, Patrick DL, de Vet HC and Terwee CB: COSMIN guideline for systematic reviews of patient-reported outcome measures. Qual Life Res 27: 1147-1157, 2018.

6. Cheung AC, Patel H, Meza-Cardona J, Cino M, Sockalingam S and Hirschfield GM: Factors that influence health-related quality of life in patients with primary Sclerosing cholangitis. Dig Dis Sci 61: 1692-1699, 2016 .

7. Klompenhouwer AJ, Sprengers D, Willemssen FE, Gaspersz MP, Ijzermans JN and De Man RA: Evidence of good prognosis of hepatocellular adenoma in post-menopausal women. J Hepatol 65: 1163-1170, 2016.

8. Onishi Y, Wakita T, Fukuhara S, Noguchi Y, Okada M, Sakaida I, Sasaki Y and Kobayashi K: Development and validation of a symptom scale specific for ascites accompanied with cirrhosis: The ASI-7. Clin Transl Gastroenterol 5: e48, 2014.

9. Schrier RW, Gross P, Gheorghiade M, Berl T, Verbalis JG, Czerwiec FS and Orlandi C; SALT Investigators: Tolvaptan, a selective oral vasopressin V2-receptor antagonist, for hyponatremia. N Engl J Med 355: 2099-2112, 2006.

10. Miyazaki T, Yamamura Y, Onogawa T, Nakamura S, Kinoshita S, Nakayama S, Fujiki $\mathrm{H}$ and Mori T: Therapeutic effects of tolvaptan, a potent, selective nonpeptide vasopressin $\mathrm{V} 2$ receptor antagonist, in rats with acute and chronic severe hyponatremia. Endocrinology 146: 3037-3043, 2005.

11. Sakaida I, Yanase M, Kobayashi Y, Yasutake T, Okada M, Okita K, Kageyama F, Miyaoka H, Nakamura H, Sakaeda H, et al; ASCITES Clinical Pharmacology Group: The pharmacokinetics and pharmacodynamics of tolvaptan in patients with liver cirrhosis with insufficient response to conventional diuretics: A multicentre, double-blind, parallel-group, phase III study. J Int Med Res 40: 2381-2393, 2012.

12. Yamamura $Y$, Nakamura $S$, Itoh $S$, Hirano $T$, Onogawa $T$, Yamashita T, Yamada Y, Tsujimae K, Aoyama M, Kotosai K, et al: OPC-41061, a highly potent human vasopressin V2-receptor antagonist: Pharmacological profile and aquaretic effect by single and multiple oral dosing in rats. J Pharmacol Exp Ther 287: 860-867, 1998.

13. Clark WF, Devuyst O and Roussel R: The vasopressin system: new insights for patients with kidney diseases: Epidemiological evidence and therapeutic perspectives. J Intern Med 282: 310-321, 2017.

14. Gülberg V, Liss I, Bilzer M, Waggershauser T, Reiser M and Gerbes AL: Improved quality of life in patients with refractory or recidivant ascites after insertion of transjugular intrahepatic portosystemic shunts. Digestion 66: 127-130, 2002.

15. Neijenhuis M, Gevers TJG, Atwell TD, Gunneson TJ, Schimek AC, Kievit W, Drenth JPH and Kamath PS: Development and validation of a patient-reported outcome measurement for symptom assessment in cirrhotic ascites. Am J Gastroenterol 113: 567-575, 2018.

16. Vilstrup H, Amodio P, Bajaj J, Cordoba J, Ferenci P, Mullen KD, Weissenborn $\mathrm{K}$ and Wong P: Hepatic encephalopathy in chronic liver disease: 2014 Practice Guideline by the American Association for the Study of Liver Diseases and the European Association for the Study of the Liver. Hepatology 60: 715-735, 2014.

17. Hiramine Y, Uojima H, Nakanishi H, Hiramatsu A, Iwamoto T, Kimura M, Kawaratani H, Terai S, Yoshiji H, Uto H, et al: Response criteria of tolvaptan for the treatment of hepatic edema. J Gastroenterol 53: 258-268, 2018.

18. Pugh RN, Murray-Lyon IM, Dawson JL, Pietroni MC and Williams R: Transection of the oesophagus for bleeding oesophageal varices. Br J Surg 60: 646-649, 1973. 
19. Moore KP, Wong F, Gines P, Bernardi M, Ochs A, Salerno F, Angeli P, Porayko M, Moreau R, Garcia-Tsao G, et al: The management of ascites in cirrhosis: Report on the consensus conference of the International Ascites Club. Hepatology 38: 258-266, 2003.

20. European Association for the Study of the Liver: EASL clinical practice guidelines on the management of ascites, spontaneous bacterial peritonitis, and hepatorenal syndrome in cirrhosis. J Hepatol 53: 397-417, 2010.

21. Iwakiri Y, Shah V and Rockey DC: Vascular pathobiology in chronic liver disease and cirrhosis - current status and future directions. J Hepatol 61: 912-924, 2014.

22. Schrier RW: Water and sodium retention in edematous disorders: Role of vasopressin and aldosterone. Am J Med 119 (Suppl 1): S47-S53, 2006

23. Wong F: The use of TIPS in chronic liver disease. Ann Hepatol 5: $5-15,2006$.
24. Bureau C, Adebayo D, Chalret de Rieu M, Elkrief L, Valla D, Peck-Radosavljevic M, McCune A, Vargas V, Simon-Talero M, Cordoba J, et al: Alfapump ${ }^{\circledR}$ system vs. large volume paracentesis for refractory ascites: A multicenter randomized controlled study. J Hepatol 67: 940-949, 2017.

25. Kawaratani H, Fukui H, Moriya K, Noguchi R, Namisaki T, Uejima M,Kitade M, Takeda K, Okura Y, Kaji K, et al: Predictive parameter of tolvaptan effectiveness in cirrhotic ascites. Hepatol Res 47: 854-861, 2017.

26. Kawaratani H, Fukui $\mathrm{H}$ and Yoshiji $\mathrm{H}$ : Treatment for cirrhotic ascites. Hepatol Res 47: 166-177, 2017.

27. Fukuhara S, Bito S, Green J, Hsiao A and Kurokawa K: Translation, adaptation, and validation of the SF-36 Health Survey for use in Japan. J Clin Epidemiol 51: 1037-1044, 1998. 